CAHIERS

MONDES

ANCIENS

\section{Cahiers « Mondes anciens »}

Histoire et anthropologie des mondes anciens

$11 \mid 2018$

La "civilisation » : critiques épistémologique et historique

\title{
La « civilisation » : critiques épistémologique et historique
}

"Civilization " : epistemological and historical critics

Michel Briand, Florence Dupont et Vivien Longhi

\section{(2) OpenEdition}

Journals

Édition électronique

URL : http://journals.openedition.org/mondesanciens/2173

DOI : $10.4000 /$ mondesanciens. 2173

ISSN : 2107-0199

Éditeur

UMR 8210 Anthropologie et Histoire des Mondes Antiques

Référence électronique

Michel Briand, Florence Dupont et Vivien Longhi, « La « civilisation » : critiques épistémologique et historique », Cahiers « Mondes anciens » [En ligne], 11 | 2018, mis en ligne le 26 mars 2018, consulté le 19 avril 2019. URL : http://journals.openedition.org/mondesanciens/2173 ; DOI : 10.4000/ mondesanciens. 2173

Ce document a été généré automatiquement le 19 avril 2019

\section{(i) $९$}

Les Cahiers "Mondes Anciens » sont mis à disposition selon les termes de la licence Creative Commons Attribution - Pas d'Utilisation Commerciale - Pas de Modification 4.0 International. 


\title{
La « civilisation » : critiques épistémologique et historique
}

\author{
"Civilization » : epistemological and historical critics \\ Michel Briand, Florence Dupont et Vivien Longhi
}

1 La journée d'étude à l'origine de cette publication était consacrée à une critique de la civilisation gréco-romaine, comme modèle, implicite ou non, de toute civilisation. Ce qui impliquait en même temps de réexaminer cette notion de civilisation, utilisée aussi bien par les enseignants et chercheurs en sciences humaines et sociales - le fameux intitulé « langue et civilisation » des cursus - que par les médias d'opinion, dont le fameux « Nous autres, civilisations, nous savons maintenant que nous sommes mortelles» de Paul Valéry, dans la Crise de l'esprit (1919), fut le prélude élégant à The Clash of Civilizations and the Remaking of World Order de Samuel P. Huntington paru en 1996.

Il n'échappe à personne qu'aujourd'hui employer le mot de civilisation dans l'espace médiatique est devenu problématique. On se souvient de Claude Guéant, alors ministre de l'Intérieur du gouvernement Fillon, (le dimanche 5 février 2012) déclarant «que toutes les civilisations ne se valent pas ", lors d'un énième débat médiatique sur le port du voile. Le Figaro avait alors demandé à quelques anthropologues pourquoi ce terme était controversé au point qu'ils évitaient soigneusement de l'utiliser depuis 50 ans et préféraient parler de cultures. François Flahault répondit que ce terme [de culture] était le plus «approprié" pour désigner «tout ce que les générations humaines se transmettent les unes aux autres de manière non biologique ». Pour Marc Crépon, «le terme de civilisation était difficilement dissociable des idéologies les plus meurtrières du $\mathrm{xx}^{\mathrm{e}}$ siècle qui avaient une idée très précise de la hiérarchie des civilisations et de leur différence de valeur. » Alfred Grosser répliquait à Claude Guéant que son jugement de valeur qui laissait supposer des hiérarchies entre civilisations sous-entendait que la civilisation islamique est inférieure à la civilisation française. Claude Guéant s'attaquait implicitement aux musulmans de France, mais la défense de Grosser est désastreuse : il est impossible de comparer la civilisation française, nationale, à une civilisation islamique, religieuse, en supposant que l'une et l'autre expressions recouvrent la moindre 
réalité. Maurice Godelier distinguait, à son tour, culture et civilisation de la façon suivante :

Contrairement à la «culture », la " civilisation » ne peut être pensée seule, car elle comporte toujours implicitement un jugement de valeur en opposition à un autre, plus barbare ; par exemple, dans « civilisation » il y a civis, c'est-à-dire citoyen. Il y a l'idée grecque et romaine que les civilisés sont ceux qui vivent dans les cités ou les États, par opposition aux barbares qui sont nomades ou paysans.

Nous voici arrivés au cœur de notre sujet. Civilisation, mot récent en français (il date du $\mathrm{XVIII}^{\mathrm{e}} \mathrm{s}$.), serait à expliquer par son étymologie latine et donc par l'idéologie grécoromaine qui opposait la civilisation des cives urbains à la barbarie des nomades. On ne reprochera pas à Maurice Godelier harcelé par un journaliste, ses approximations historiques; on peut, au contraire, lui savoir gré d'avoir rappelé la place que la civilisation gréco-romaine tient dans l'idéologie contemporaine. La notion de civilisation nous viendrait de l'Antiquité. Donc, pour certains, la civilisation gréco-romaine serait au début et à l'origine de l'humanité civilisée, et pour d'autres, c'est d'elle que viendrait le narcissisme méprisant de la civilisation européenne. Les débuts de l'anthropologie moderne au XIX ${ }^{\mathrm{e}}$ s. sont marqués par de tels jugements de valeur. Dans Ancient Society (1877), Lewis Morgan affirme que l'humanité évolue en passant par trois stades successifs : la sauvagerie, la barbarie et la civilisation. Les plus civilisés étaient, selon lui, les Américains. Les Européens l'étaient moins car ils conservaient encore des vestiges féodaux.

Ces commentaires autour de «l'affaire Guéant » montrent que la notion de civilisation est aussi floue qu'explosive. Notion qui de loin semble évidente, la civilisation s'éparpille en sens divers quand on utilise le mot. Seul repère solide, la référence à l'Antiquité. Miracle grec ou péché originel, la civilisation gréco-romaine surgit dès qu'il est question de civilisation.

5 Peut-on trouver à la notion de civilisation un statut épistémologique? N'est-elle pas définitivement écrasée sous ses origines gréco-romaines? La notion anthropologique de culture, prééminente depuis quelques décennies, ne serait-elle pas d'un meilleur usage?

L'étude liminaire de Claude Calame, "Civilisation et Kultur: de Friedrich August Wolf à Sigmund Freud», propose des réponses à ces questions. Chez Wolf, historien de la littérature antique initiateur de ce qui deviendra la Klassische Philologie, les Grecs se distinguent comme un peuple disposant d'une « culture de l'esprit » ${ }^{1}$. Cette Kultur permet de différencier les Grecs, les Romains et leurs successeurs allemands des autres « civilisations ». La culture gréco-latine lui permet donc de classer les civilisations. De telles conceptions se retrouvent dans ce que Calame appelle de «nouveaux avatars du "Grand partage" ", chez un historien des religions contemporain comme J. Assman par exemple, qui produit une opposition entre civilisation religieuse de l'écrit et autres cultures religieuses orales ${ }^{2}$. Or, Calame montre que la religion des Grecs ne se laisse pas comprendre dans ce partage ${ }^{3}$. Des notions de Kultur/ «civilisation» plus critiques pourraient guider la réflexion des anthropologues de l'antiquité, dans le sillage de celle que Freud a développée dans son Malaise dans la civilisation, œuvre sur laquelle revient Claude Calame ${ }^{4}$. On peut sans doute interroger la formation de l'individu dans la civilisation, c'est-à-dire à travers des réseaux de sociabilité et de normes. Cela revient en fait à penser des civilisations en leur donnant, au cas par cas, un statut épistémologique dans l'analyse des processus de fabrication de l'individu dans une collectivité. La civilisation, dont on prétend trouver la source dans l'antiquité, fausse donc profondément 
la compréhension qu'on peut avoir de ces mêmes mondes anciens. La notion, si l'on tient à la conserver, ne pourrait être utile que défaite, vidée de son sens évolutionniste, et resémantisée dans une perspective anthropologique.

7 Il fallait donc reprendre la question au début et faire l'archéologie de la notion. Rappeler d'abord que la notion et le terme sont modernes, comme le développe et le précise Jan Blanc au début de son article. Ce mot apparaît pour la première fois sous la plume du Marquis de Mirabeau, le père, en $1756^{5}$. Il remplace civilité. Émile Benveniste écrit :

Pour Mirabeau, la civilisation est un procès que l'on dénommait jusqu'alors «police», un acte tendant à rendre l'homme et la société plus "policés», l'effort pour amener l'individu à observer spontanément les règles de la bienséance et pour transformer dans le sens d'une plus grande urbanité les mœurs de la sociétét.

L'Encyclopédie offre un bon exemple de ce lien primordial de la notion de civilisation à l'antiquité. Il n'y a encore que très peu d'occurrences du mot civilisation dans l' Encyclopédie de Diderot et d'Alembert. Deux usages, au sens moderne, viennent à l'occasion d'une réflexion sur les Vies de Plutarque, (art. « Vies » et "Zones tempérées » rédigés par Louis de Jaucourt). L'auteur égrène les héros civilisateurs de la Grèce ancienne, Socrate, Solon, Lycurgue, etc. :

Il [Plutarque] me fait converser délicieusement dans ma retraite gaie, saine et solitaire, avec ces morts illustres, ces sages de l'antiquité révérés comme des dieux, bienfaisans comme eux, héros donnés à l'humanité pour le bonheur des arts, des armes et de la civilisation.

Benveniste prend acte que l'on passe d'une notion d'état, la police des mœurs, à une notion d'action : la civilisation va avec l'idée de progrès moral, technique ou autre. Il n'est pas étonnant que cette mutation ait eu lieu au $\mathrm{XVIII}^{\mathrm{e}} \mathrm{s}$. et que la notion de civilisation s'installe largement au XIX ${ }^{e}$ et début du $\mathrm{Xx}^{\mathrm{e}}$ siècle, en même temps que la colonisation qui apportait aux «sauvages» les «bienfaits de la civilisation». Les Grecs et les Romains n'ont rien à voir avec ce mot qui ne correspond ni à philanthropia, ni à humanitas, ni à cultus, et pas plus à civilis.

10 C'est pourquoi dans un premier temps nous avons demandé à plusieurs chercheurs de faire l'archéologie de la "civilisation gréco-romaine», telle que nous la connaissons aujourd'hui dans les ouvrages savants comme dans les jeux vidéo.

11 Blaise Dufal propose une enquête sur les usages historiographiques de la notion dans un article intitulé: "Le fantasme de la perfection originelle. La Grèce antique comme matrice du modèle civilisationnel.» Dans les manuels d'histoire et ouvrages de vulgarisation et chez des classiques de l'historiographie française $\mathrm{du} \mathrm{xx}^{\mathrm{e}} \mathrm{s}$., on voit que la "civilisation", faute de définition rigoureuse, n'est pas un concept scientifique. Elle produit une vision idéologique de la culture et de l'histoire, fondée sur un fantasme de la Grèce antique, idéalisée depuis le XVIII ${ }^{\mathrm{e}} \mathrm{s}$. Les Grecs de l'Antiquité, dont la modernité européenne se veut l'héritière, seraient ainsi l'origine géniale et les exemples parfaits de la science, des arts et de la politique.

12 Jan Blanc déplace la question sur le terrain de l'histoire de l'art à la période moderne. Il interroge le «problème de la civilisation grecque » chez Winckelman. Johann Joachim Winckelmann (1717-1768) est à l'origine du " miracle grec », cette vision de la civilisation grecque comme un âge d'or politique, moral et artistique. Il parle certes d'Antiquité grecque et non de civilisation, le mot n'existe pas encore au sens moderne, mais les deux livres, qu'il a consacrés à l'Antiquité grecque, l'étudient comme un monde dont il s'agit de rendre compte des grands principes à travers l'étude de ses œuvres d'art. C'est ainsi qu'il 
décrit la Grèce en faisant de sa supériorité artistique un a priori. Winckelmann commence à écrire sur l'art antique sans avoir vu la moindre statue. Ses écrits sont et resteront des constructions imaginaires, déduites de cet a priori. La Grèce est pour lui un mythe. La Grèce est, pour Winckelmann, la seule civilisation qui, dans l'histoire, soit parvenue à s'arracher à la barbarie de la simple nature sans être touchée par la corruption des mœurs, processus inhérent à la culture. Mais ce miracle fut éphémère et a disparu à jamais. Après la perfection de l'art classique au v $v^{e} s$., la Grèce a été entraînée dans une décadence irréversible. L'histoire de Winckelmann est donc téléologique, parce qu'elle pose a priori la grandeur suprême de l'art grec. Mais elle est également eschatologique, dans la mesure où le "grand style » est irrémédiablement perdu. La "civilisation grecque » n'est pas, pour lui, une " période » de l'histoire mais, plutôt, une utopie servant aux Modernes à se raconter, en construisant, dans le temps et le passé, l'origine d'une grandeur perdue dont ils ne peuvent nullement être considérés comme les enfants ou les héritiers, mais qu'ils doivent apprendre à regretter. La civilisation grecque telle qu'elle est inventée par Winckelmann tient donc des deux acceptions modernes de cette notion. Celle d'un progrès, mais qui n'est observable que dans les restes et les ruines du passé et celle d'une décadence inéluctable.

La culture ludique contemporaine permet aussi de voir le lien étroit de l'antiquité à la notion de civilisation. Dans « Alexandre et Octavien contre Bismarck et Gengis Khan. Les usages (problématiques) de l'Antiquité gréco-romaine dans l'univers ludique de Civilization ", Emmanuelle Valette s'intéresse au jeu vidéo à succès Civilization, réédité et amélioré plusieurs fois depuis 1991 jusqu'à son ultime version de 2016. La durée de son succès international en fait un bon témoin de certaines idées populaires contemporaines sur la notion de civilisation. Le joueur peut choisir de développer une civilisation, dont les critères de définition sont d'ailleurs problématiques, parmi plusieurs, sans hiérarchie $a$ priori entre elles. Au centre du jeu, il y a l'habileté du joueur et sa capacité à faire évoluer sa civilisation. La victoire viendra de l'inventivité technique qu'il aura su insuffler à celle qu'il aura prise en main. Le cours du jeu suit un « évolutionnisme et un ethnocentrisme décomplexés" puisqu'une civilisation avance vers la domination mondiale à coup d'inventions technologiques successives, en construisant aussi de fortes et grandes cités. Si toutes les civilisations sont ainsi calquées sur un modèle occidental, les mondes anciens ont toutefois un lien encore plus étroit à $L a$ civilisation. Les civilisations antiques apparaissent comme "originelles» et sont permanentes dans l'offre du jeu, comme incontournables, alors que d'autres (Iroquois, Zoulous...) sont des options qui disparaissent ou reparaissent au fil des versions. La Grèce et Rome disposent aussi d'un certain nombre de traits spécifiques et d'atouts technologiques discrets qui en font des civilisations d'élection pour les habitués. Par ailleurs la culture antique irrigue l'ensemble du processus d'évolution inventive : les atouts culturels (les «merveilles» du monde par exemple) et les innovations que peut développer telle ou telle civilisation choisie par le « gamer » sont souvent pensés en référence aux langues ou cultures grecques et romaines. L'antiquité proposée n'est donc pas un monde ludique comme un autre ou un simple facteur d'exotisme : elle est essentielle à l'imaginaire de la civilisation elle-même. quelle aurait civilisé l'humanité, en ayant inventé des formes culturelles devenues le patrimoine de l'humanité, parce qu'elle aurait anticipé sur la modernité. Ces « inventions » jusqu'à celle de la notion même d'« invention ", sont en fait des inventions de notre modernité, comme le montrent les cinq analyses suivantes. 

d'imaginaire; tel est le cas de "l'enthousiasme ", comme le montre Michel Briand, dans son article «L'invention de l'enthousiasme poétique ». L'enthousiasme poétique est une invention moderne, créant une illusion rétrospective. Les modernes, qui opposent improvisation inspirée et technique d'écriture, attribuent aux poètes grecs archaïques et classiques un rapport privilégié avec le divin, l'inspiration; ils auraient chanté, possédés par une fureur mystique : « le dieu était en eux ». Or pour les Grecs les aèdes étaient à la fois aimés des Muses et artisans de vers. Une archéologie des mots enthéos, enthousiasmos, s'imposait. L'enquête philologique montre que le sens d'enthéos n'est pas celui qu'une tradition étymologique lui donne, par une interprétation possessive - locative de l'adjectif enthéos. L'adjectif enthéos peut être l'équivalent emphatique de theios, et signifier «très divin». L'inspiration poétique sous l'effet de l'intériorisation d'un souffle transcendant, par laquelle le poète-prophète a «un dieu en lui », vient (relu par l'antiquité tardive et certains modernes) directement de Platon, qui a comme souvent joué avec les mots et rapproché mantis (la divination) de mania (la folie) et inventé une figure du poéte-prophète inspiré. Cette inspiration prophétique réinterprétée par les néoplatoniciens se retrouve chez certains mystiques chrétiens ou au contraire chez certains critiques du paganisme. La reconstruction moderne de l'inspiration grecque oppose écriture et oralité comme une alternative radicale, projetant sur l'histoire de la poésie grecque le grand partage constitutif de la modernité depuis l'âge romantique.

La notion de personne charrie avec elle tout un imaginaire occidental philosophique, juridique et religieux du progrès de la conscience. Florence Dupont en critique la prétendue invention par les Romains. Cette idée souvent reprise a notamment été soutenue par M. Mauss dans « Une catégorie de l'esprit humain : la notion de personne ». Or, le raisonnement de Mauss n'est pas une démonstration scientifique et repose sur une pensée a priori de la place dominante de Rome dans la civilisation occidentale. Sous l'apparence d'une enquête portant sur des faits sociaux, juridiques, et religieux, c'est en fait principalement l'hypothèse d'une évolution sémantique du mot persona qui sous-tend l'exposé de Mauss : le «masque rituel » archaïque des ancêtres deviendrait la «personne juridique » du droit romain, définitivement inventée à la période classique. Le savant superpose en fait, «dans un coup de force sémantique », les sens d'imago et de persona : il n'y a aucune raison probante de penser que la persona était un masque rituel d'ancêtre au même titre que l'imago. Quant à la notion juridique de persona, elle ne renvoie pas non plus à un ensemble de droits liés à la "personne ", mais plutôt à un rôle temporaire pris dans un procès. La «personne» ne se trouve donc pas déjà dans la persona, et la dynamique historique d'une invention romaine de la personne voulue par Mauss disparaît du même coup. D'autres stratégies pour sauver l'invention de la personne se laissent voir : l'essentialisation de la notion avant toute enquête philologique préalable ou encore l'utilisation de catégories modernes préconstruites. Elles ne laissent pas de surprendre chez un savant de cette ampleur. Quelles sont les causes possibles de ce discours fictionnel sur l'« invention » antique, dans le contexte de travail qui a été celui de l'ethnologue? En posant cette question Florence Dupont ouvre la voie à une critique pragmatique du recours à la notion d'invention chez les antiquisants.

17 L'histoire de la médecine n'est pas avare non plus d'« inventions », et les Grecs, avec leur légendaire figure d'Hippocrate, ont une large part dans ce grand récit, comme cherche à le montrer Vivien Longhi dans un article intitulé : « Hippocrate a-t-il inventé la médecine d'observation? ». Les traités de la «médecine hippocratique », par exemple Épidémies I-III 
et Pronostic, présentent des relevés de signes pathologiques apparemment scrupuleux, où le corps malade serait doté de sens par un médecin expert du pronostic. Au XVIII $\mathrm{s}$., médecins et professeurs y voient les fondements de leur médecine d'observation, fille de la clinique, alors qu'il s'agit de textes largement spéculatifs. Une approche pragmatique du regard médical ancien dégagerait pourtant la médecine grecque même, travestie par la notion moderne d'observation.

Dans le domaine de l'histoire littéraire s'érigent et pèsent encore sur les Grecs d'autres « inventions ». Marie Saint-Martin, dans son article intitulé "L'invention de la tragédie selon Pierre Brumoy: de quelques pièges du relativisme" s'intéresse aux réflexions modernes de P. Brumoy sur la tragédie (1730). La recherche des « inventeurs » du théâtre classique conduit l'auteur à un certain nombre d'apories ou de thèses paradoxales. Eschyle et Homère sont aussi bien l'un que l'autre considérés comme ses inventeurs. Les auteurs épiques et tragiques grecs semblent avoir toujours été aristotéliciens. Si les Grecs ainsi compris sont à l'origine du théâtre classique, comment expliquer alors que leurs pièces ne soient plus appréciées sur la scène française ? La force originelle créatrice des anciens doit être reprise, cultivée et amendée par les modernes. Conserver les beautés universelles des anciens, mais en gommant et lissant leur barbarie et leur brutalité. Il faut une civilisation de la civilisation première, pourrait-on dire en jouant sur les mots. Après ce travail de polissage le lien doit se rétablir entre la civilisation grecque et les nations policées, au premier chef desquelles la nation française. L'histoire de «l'invention » de la tragédie par les Anciens sert donc à unir entre elles des nations culturellement supérieures.

La notion même d'invention finit par poser problème, d'autant qu'elle reste utilisée chez ceux-là mêmes qui sembleraient devoir la contester, comme le montre Anne-Gabrielle Wersinger dans « L'invention de l'invention : archéologie ou idéologie? ».

En sciences humaines, on constate l'inflation des titres mentionnant le mot ambigu d'invention. Et même si l'anthropologie prétend en avoir fini avec « les inventeurs grecs » et l'archéologie du "Miracle grec », Gernet et Vernant ne se sont pas entièrement défaits d'une interprétation démiurgique et progressiste de l'histoire. Et malgré l'autoréférentialité de l'anthropologie de Loraux ou Detienne, la critique des « idéologies » résiste mal au paradigme prométhéen de l'innovation, qui s'impose dans l'institution de la recherche contemporaine.

21 Cette dernière étude notamment, en épilogue provisoire des précédentes, montre qu'il reste à repérer explicitement d'autres "inventions ", qui seraient à soumettre à une généalogie philologique, épistémologique, historiographique, critique, en même temps qu'à l'étude précise de leurs usages idéologiques les plus contemporains. C'est à une réflexion générale qu'on invite ici, sur le rôle accordé, voire imposé, aux références antiques, en particulier aux notions et catégories, comme celles de civilisation et d'invention, dans les sciences humaines et sociales, et d'autre part sur la valeur de critique radicale que peut avoir l'étude même de l'Antiquité, pour nos catégories contemporaines les plus évidentes. 


\section{NOTES}

1. F. A. Wolf, Darstellung der Althertumswissenschaft nach Begriff, Umfang, Zweck und Werth, Museum der Alterthums-Wissenschaft 1, Berlin, 1807, p. 10-142.

2. J. Assmann, Religion und Kulturelles Gedächtnis. Zehn Studien, Munich, 2000 ; trad. anglaise : Stanford, 2005.

3. C. Calame, Qu'est-ce que la Mythologie grecque ?, Paris, 2015.

4. Édition originale : S. Freud, Das Unbehagen in der Kultur, Vienne, 1930.

5. Voir l'étymologie de « civilisation » sur le site du CNRTL (Centre National de Ressources Textuelles et Lexicales) à l'adresse : http://www.cnrtl.fr/etymologie/civilisation

6. Émile Benveniste, Problèmes de linguistique générale, I, Paris, 1966, p. 336-345.

\section{AUTEURS}

\section{MICHEL BRIAND}

Université de Poitiers

\section{FLORENCE DUPONT}

Université Paris Diderot-Paris 7

\section{VIVIEN LONGHI}

Université Lille 3 